Supporting Information for

\title{
Crystal Engineering of Amphiphilic Organic Dye for Metallic Coloration
}

\author{
Seok-In Lim, ${ }^{a}$ Ki-Hyun Ryu, ${ }^{a, b}$ Dae-Young Jeon, ${ }^{b}$ Cheol-Min Yang, ${ }^{b}$ Luciano De Sio, ${ }^{c}$ \\ Dae-Yoon Kim, ${ }^{*, b}$ and Kwang-Un Jeong ${ }^{*, a}$
}

\begin{abstract}
${ }^{a}$ Department of Polymer-Nano Science and Technology, Department of Nanoconvergence Engineering, Jeonbuk National University, Jeonbuk 54896, Korea.

${ }^{\mathrm{b}}$ Institute of Advanced Composite Materials, Korea Institute of Science and Technology, Jeonbuk 55324, Korea.

${ }^{\mathrm{c}}$ Department of Medico-Surgical Sciences and Biotechnologies, Sapienza University of Rome, Roma 00185 , Italy.
\end{abstract}

*kujeong@jbnu.ac.kr (K.-U. Jeong) and kdaeyoon@kist.re.kr (D.-Y. Kim)

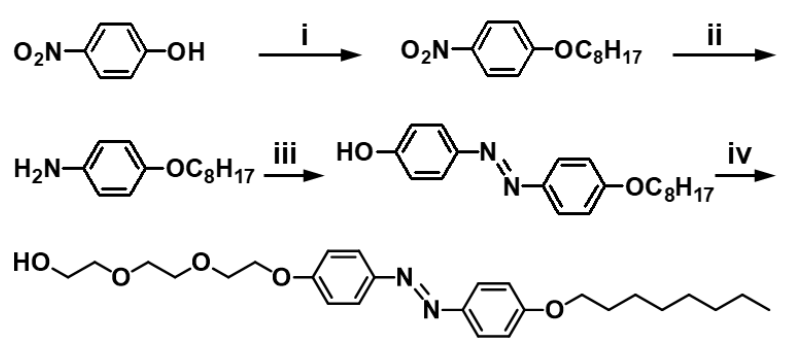

Figure S1. Synthetic routes of $\mathrm{A}_{1} \mathrm{GC}$ compounds. Reagents and conditions: i) Bromooctane, potassium carbonate, dimethylformamide, $80^{\circ} \mathrm{C}, 24 \mathrm{~h}$; ii) Raney nickel catalyst, hydrazine monohydrate, ethanol, $45^{\circ} \mathrm{C}, 6 \mathrm{~h}$; iii) Hydrochloric acid, sodium nitrite, sodium hydroxide, phenol, $0^{\circ} \mathrm{C}, 1 \mathrm{~h}$; iv) Triethylene glycol monochlorohydrin, potassium carbonate, dimethylformamide, $80{ }^{\circ} \mathrm{C}, 24 \mathrm{~h}$. 

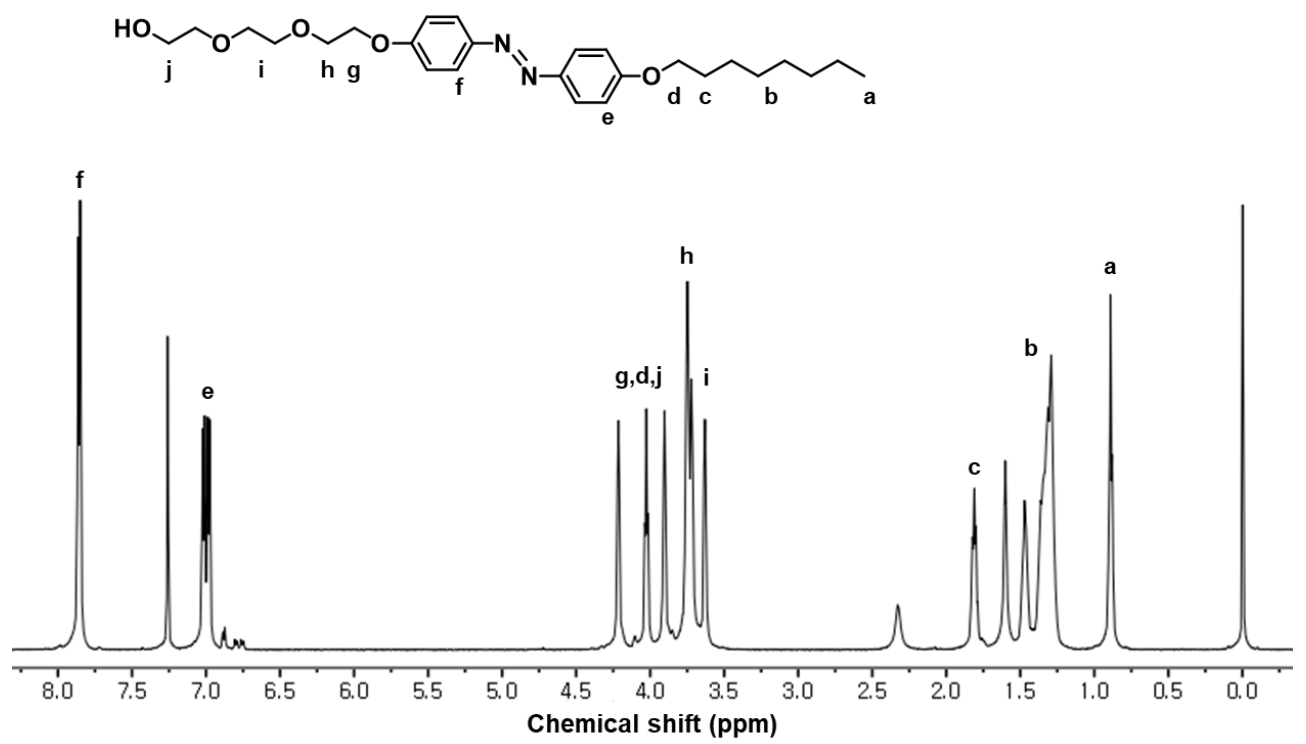

Figure S2. ${ }^{1} \mathrm{H}$ NMR spectra of $\mathrm{A}_{1} \mathrm{GC}$ compound.
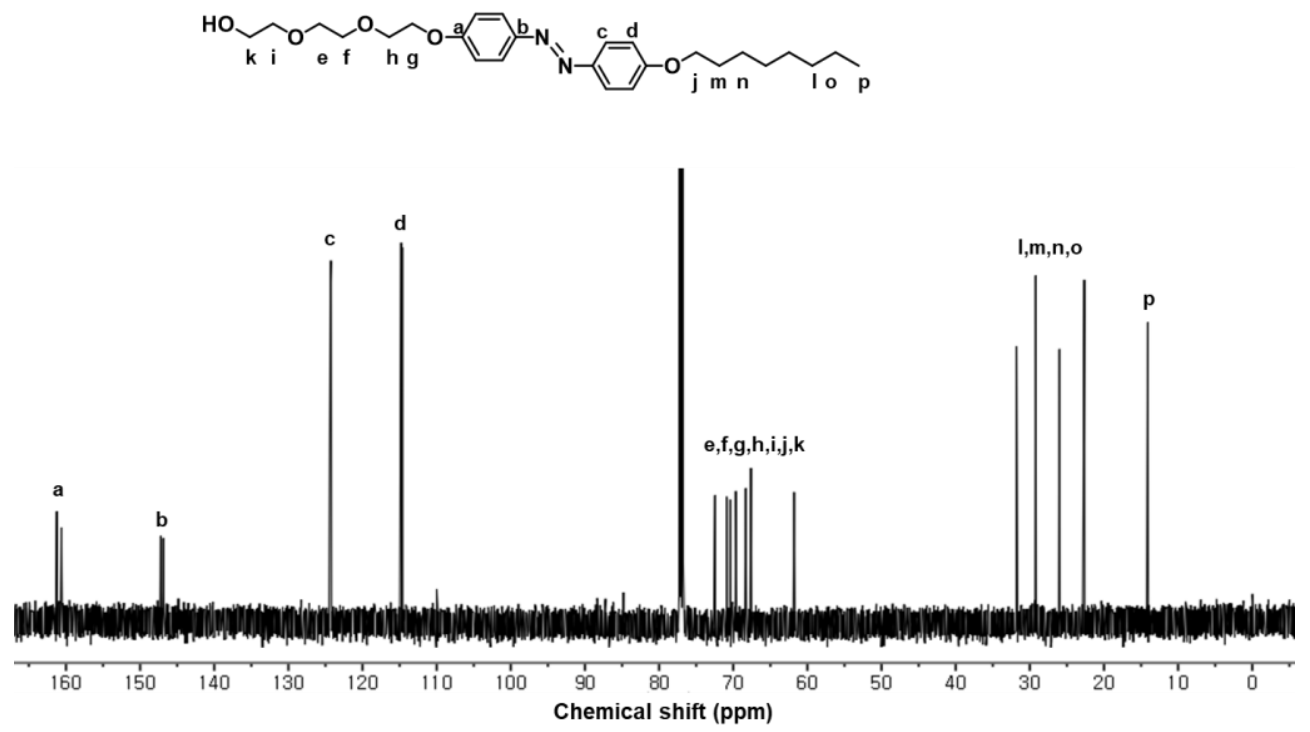

Figure S3. ${ }^{13} \mathrm{C}$ NMR spectra of $\mathrm{A}_{1} \mathrm{GC}$ compound. 


\begin{tabular}{ccc}
\hline & $\begin{array}{c}\text { Experimental } \\
\text { Content (\%) }\end{array}$ & $\begin{array}{c}\text { Calculated } \\
\text { Content (\%) }\end{array}$ \\
\hline $\mathrm{C}$ & 68.52 & \\
& 68.37 & 68.10 \\
& 68.19 & \\
$\mathrm{H}$ & 8.362 & \\
& 8.359 & \\
& 8.331 & \\
$\mathrm{~N}$ & 6.103 & 6.11 \\
& 6.096 & \\
\hline
\end{tabular}

Figure S4. EM analysis of $\mathrm{A}_{1} \mathrm{GC}$ compound.
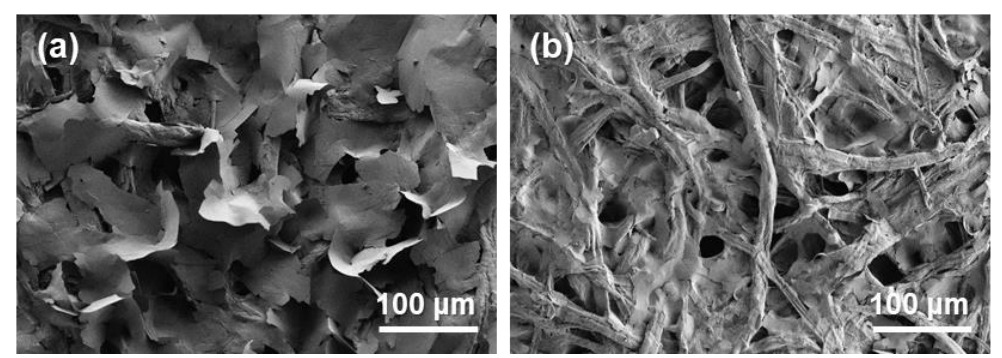

Figure S5. SEM images for $\mathrm{A}_{1} \mathrm{GC}$ coated side (a) and cellulose mat side (b) of bilayer films. 
(a)

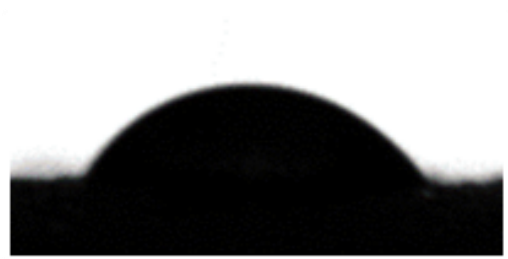

(b)

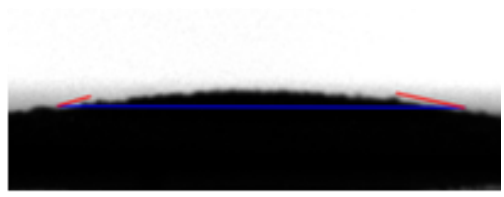

Figure S6. WCA for $\mathrm{A}_{1} \mathrm{GC}$ coated side (a) and cellulose mat side (b) of bilayer films.

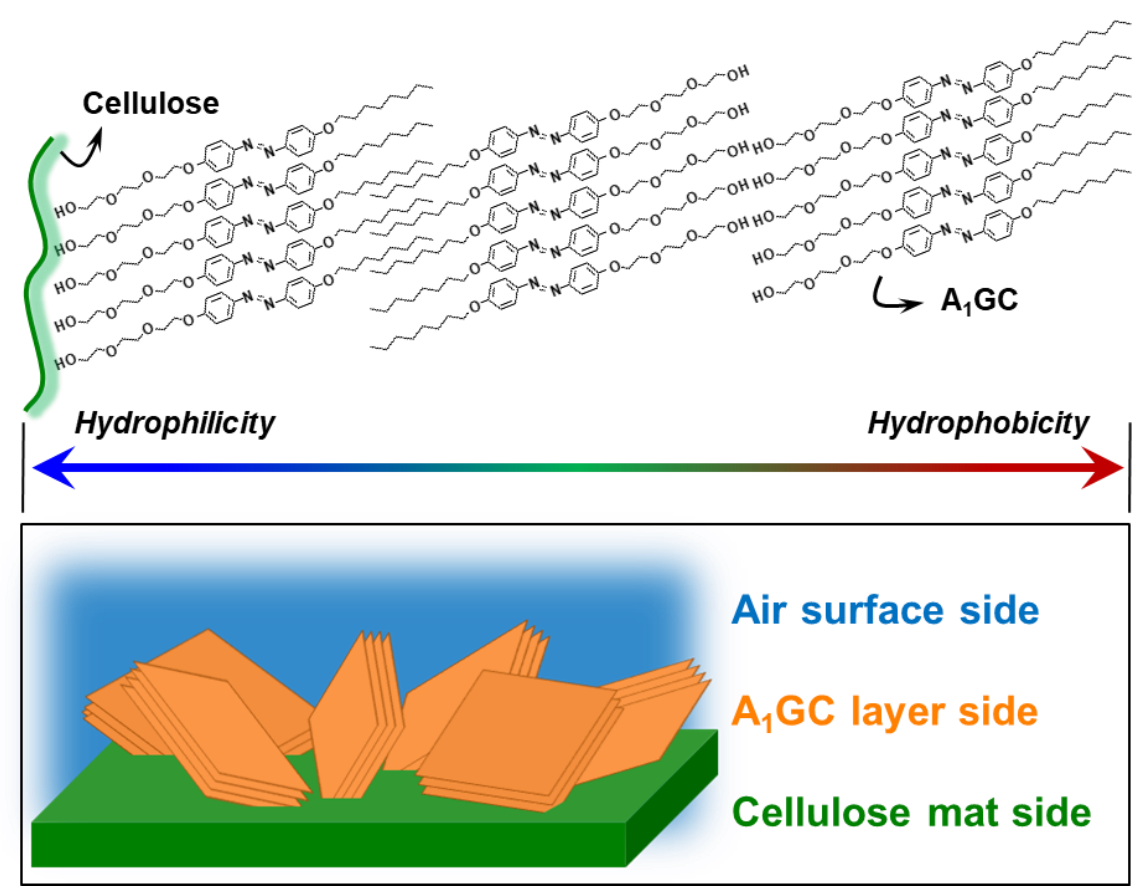

Figure S7. Schematic illustration of the composite film fabricated by filtration of $\mathrm{A}_{1} \mathrm{GC}$ crystal in the ethanol onto the filter paper. 
(a)

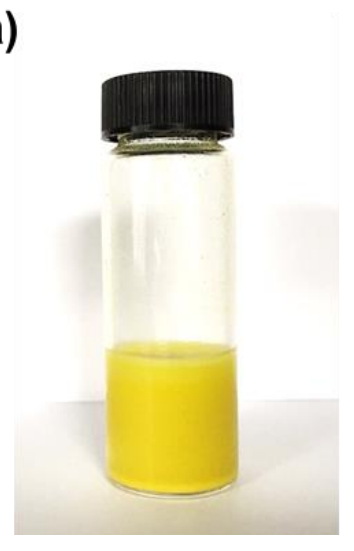

(b)

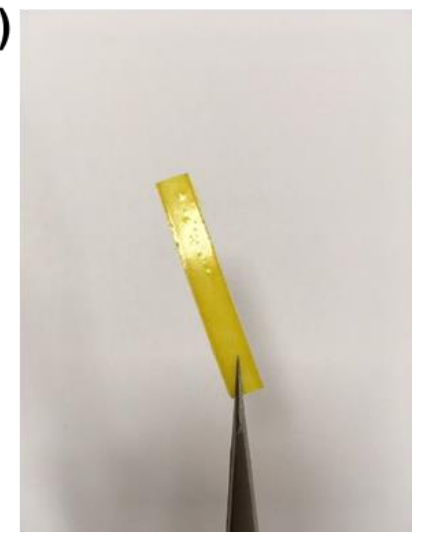

Figure S8. $A_{1}$ GC solution with PVA in water (a) and free-standing gold color films (b).
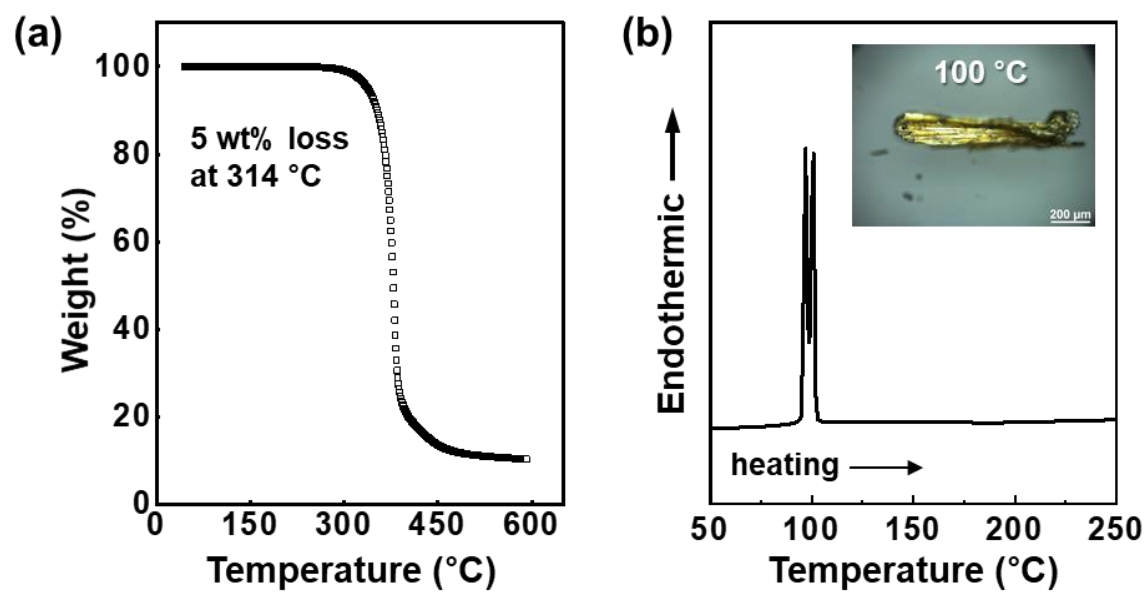

Figure S9. TGA (a) and DSC (b) thermograms of $\mathrm{A}_{1} \mathrm{GC}$ crystal, and its microphotograph at $100{ }^{\circ} \mathrm{C}$ (inset). 


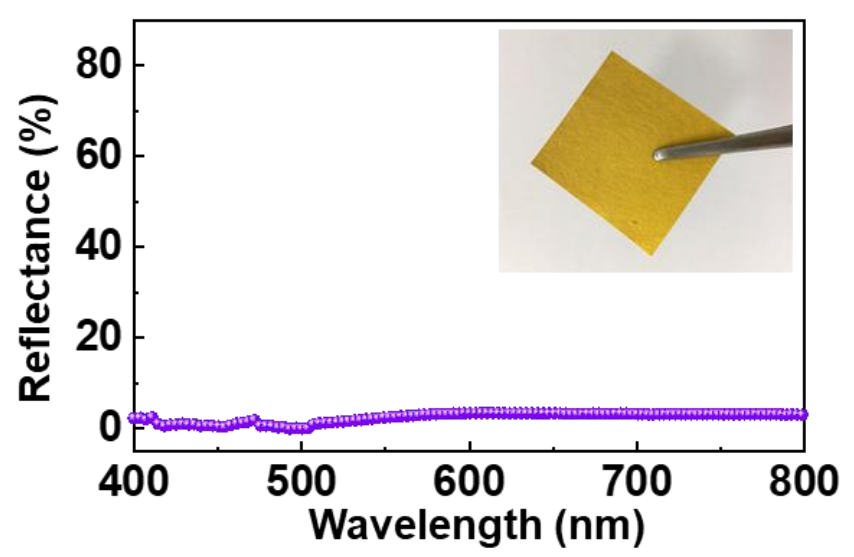

Figure S10. Reflectance of film made by $\mathrm{A}_{1} \mathrm{GC}$ powder.

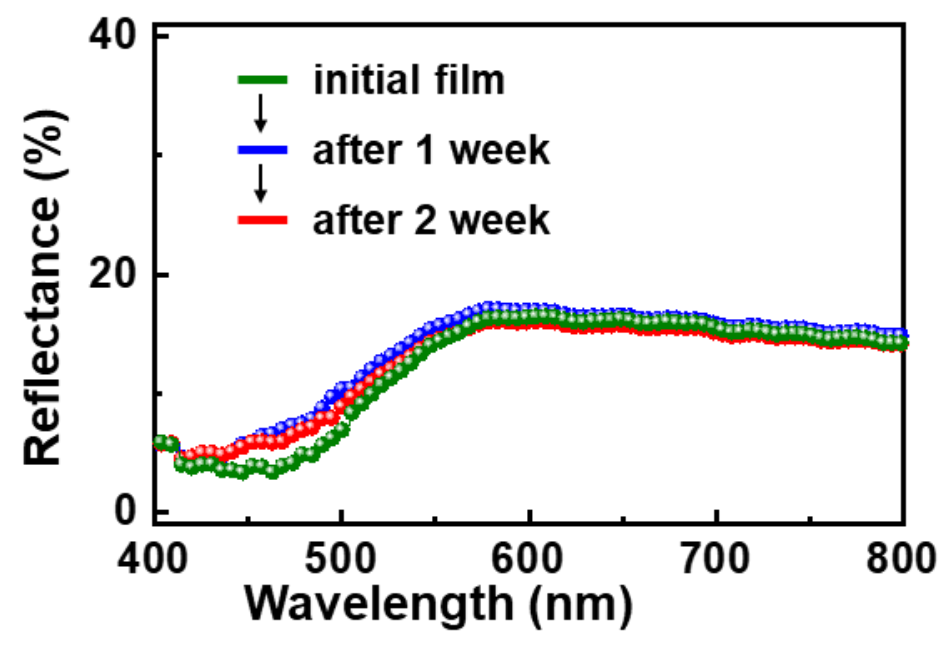

Figure S11. Reflectance of $\mathrm{A}_{1} \mathrm{GC}$ crystal before and after light irradiation. 


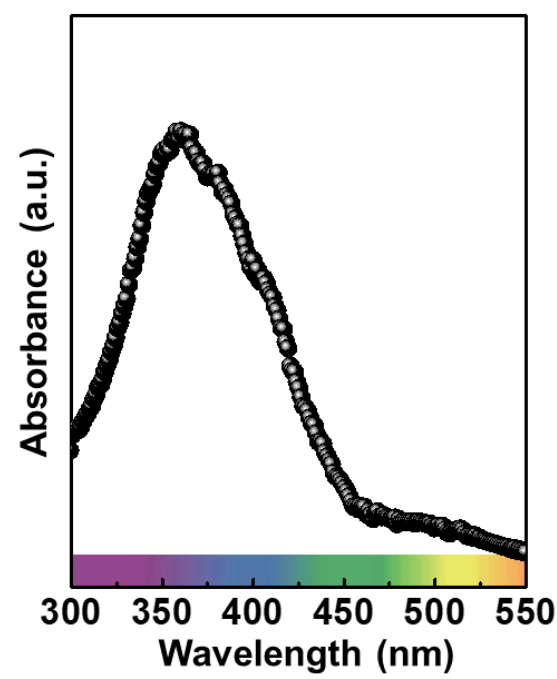

Figure S12. Absorption property of $\mathrm{A}_{1} \mathrm{GC}$ crystal.

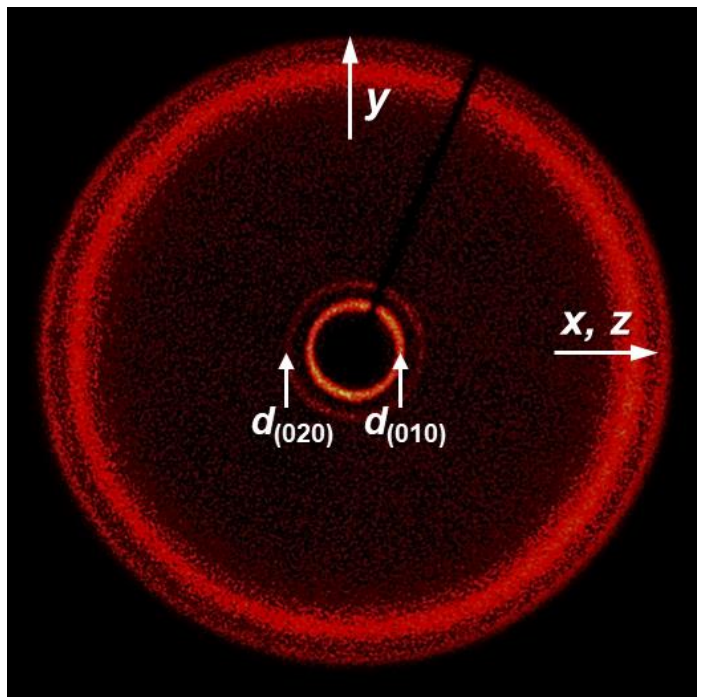

Figure S13. 2D WAXD image of $\mathrm{A}_{1} \mathrm{GC}$ powder. 


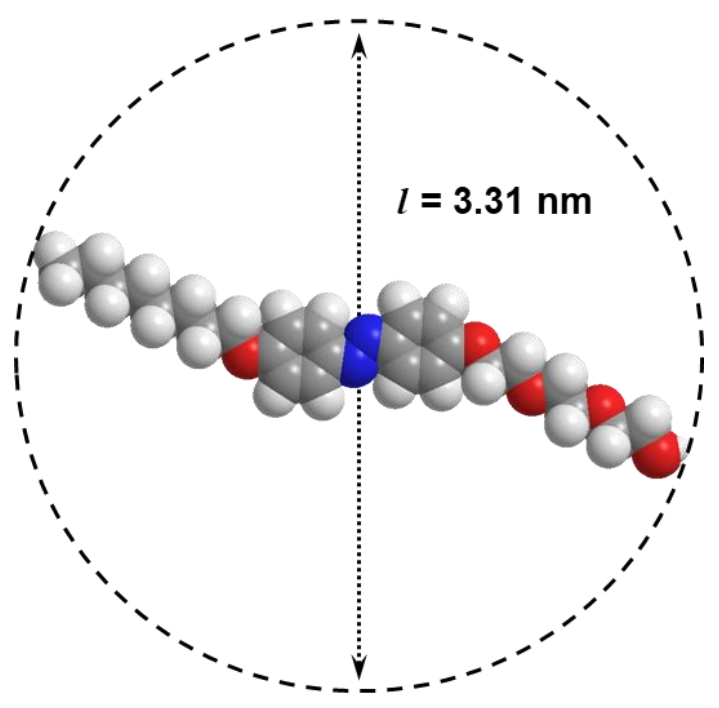

Figure S14. Calculated molecular geometry of $\mathrm{A}_{1} \mathrm{GC}$ molecule.

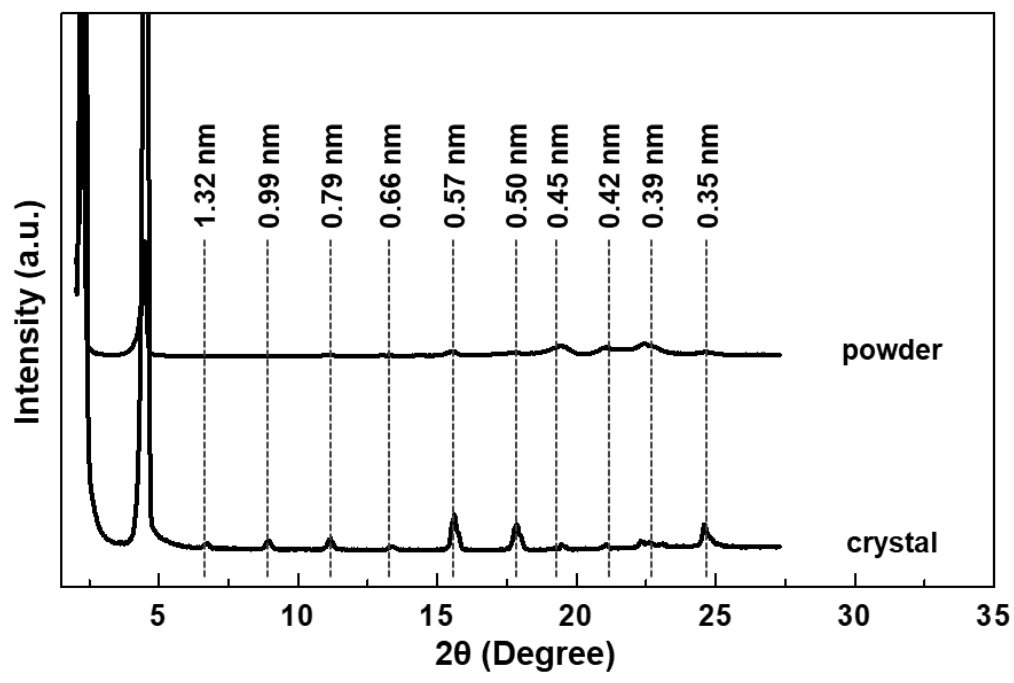

Figure S15. 1D WAXD patterns of $\mathrm{A}_{1} \mathrm{GC}$ powder and crystal. 


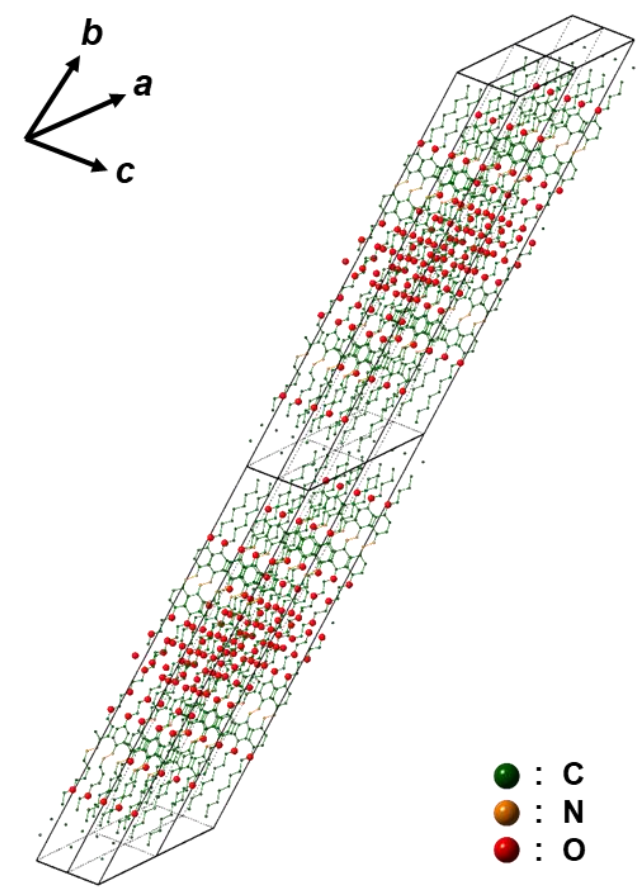

Figure S16. Monoclinic lattice of $A_{1} G C$ molecule.

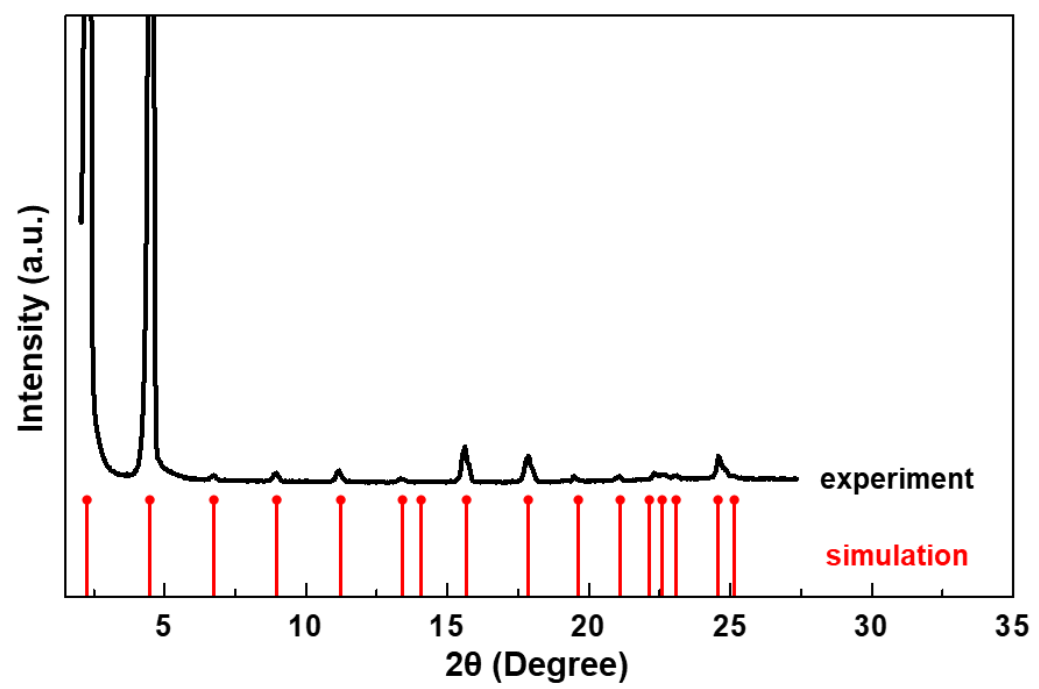

Figure S17. Experimental 1D WAXD results of $A_{1} G C$ and its simulated pattern. 